\title{
Estudio físico-químicos de los huevos de Iguana iguana Linnaeus, 1758
}

\section{Physical-chemical study of Iguana iguana Linnaeus, 1758 eggs}

\author{
Margarita Guzmán ${ }^{1 *}$ Ph.D; Herly Zuñiga²; John Moreno S² M.Sc; \\ Jairo Granados M² M.Sc.
}

${ }^{1}$ Universidad de Buenos Aires, Argentina. 2Universidad De Cundinamarca Facultad de Ciencias Agropecuarias: Fusagasuga, Colombia.

\section{KEYWORDS:}

Egg;

Iguana iguana;

physical characterization;

chemical composition.

\section{ABSTRACT}

Iguana iguana eggs were physically and chemically characterized. the physical tests were performed by methods of spectrophotolorimetry, calibrator, tape measure, micrometer, flexometer and flotation and chemical tests according to the guidelines required by A.O.A.C. The results obtained showed with respect to the physical characteristics: On average, a weight of $14.78(\mathrm{~g})$; longitudinal diameter $39.70 \mathrm{~mm}( \pm 0.49)$, cross-sectional diameter $26.41 \mathrm{~mm}( \pm 0.6)$, perimeter length $11.55 \mathrm{~cm}( \pm 0.2)$, perimeter width $9.07 \mathrm{~cm}( \pm 02)$, among other characteristics evaluated. Regarding the chemical composition, in shell $51.23 \%( \pm 3.02)$ Inorganic Calcium; $20.17 \%( \pm 1.56)$ Inorganic Phosphorus; in albumen $51.47 \%( \pm 1.05)$ of crude protein, $17.02 \%( \pm 1.33)$ of raw fat and in compact albumen a weight of $3.83 \mathrm{~g}( \pm 0.21)$. Through multiple regressions, it was determined that the weight depends on the percentage of shell and the compact albumen in $87 \%$. The specific gravity depends not only on the perimeter length but also on the percentage of shell and the thickness of this in the equatorial zone in a $86.3 \%$. The percentage of shell, weight, specific gravity and thickness in the equatorial zone in $88.3 \%$. The chromatic characterization determined its color between medium yellow and yellowish white.

\section{RESUMEN}

Huevo;

Iguana iguana; caracterización física; composición química.

INFORMACIÓN Recibido: 10-01-2018; Aceptado: 22-06-2018. Publicado: 02-07-2018 Correspondencia autor: marguzman@agro.uba.ar
Caracterización físico-químicamente de huevos de Iguana iguana. Las pruebas físicas se realizaron por métodos de Espectrofotocolorimetría, calibrador, cinta métrica, micrómetro, flexómetro y flotación y las pruebas químicas acorde a los lineamientos exigidos por la A.O.A.C. Los resultados obtenidos mostraron con respecto a las características físicas: En promedio, un peso de $14,78(\mathrm{~g})$; diámetro longitudinal $39,70 \mathrm{~mm}( \pm 0,49)$, diámetro transversal $26,41 \mathrm{~mm}( \pm 0,6)$, largo perimetral $11,55 \mathrm{~cm}( \pm 0,2)$, ancho perimetral $9,07 \mathrm{~cm}( \pm 0,2)$, entre otras caractericticas evaluadas. Respecto a la composición química, en cáscara $51,23 \%( \pm 3,02)$ Calcio Inorgánico; $20,17 \%( \pm 1,56)$ Fósforo Inorgánico; en albumen $51,47 \%( \pm 1,05)$ de proteína bruta, un $17,02 \%( \pm 1,33)$ de grasa cruda y en albumen compacto un peso de $3,83 \mathrm{~g}( \pm 0,21)$. Mediante regresiones múltiples se determinó que el peso depende del porcentaje de cáscara y del albumen compacto en un $87 \%$. La gravedad específica depende no solo del largo perimetral sino también del porcentaje de cáscara y del grosor de ésta en la zona ecuatorial en un $86,3 \%$. El porcentaje de cáscara, del peso, gravedad específica y grosor en la zona ecuatorial en $88,3 \%$. La caracterización cromática determinó su color entre amarillo medio y blanco amarillento. 
Guzmán et al - Estudio físico-químicos de los huevos de Iguana iguana Linnaeus, 1758.

\section{INTRODUCCIÓN}

La iguana verde es conocida con el nombre científico de Iguana iguana, es un reptil de hábitos arbóreos, diurno, herbívoro, originario de los bosques secos y bosques húmedos tropicales de América Latina (CITES, 2012). Es una de las especies de iguánidos más grandes que existen, pueden alcanzar hasta $2 \mathrm{~m}$ de longitud total (VALENZUELA, 1981). Presenta cabeza ancha, hocico redondeado, con dientes pleurodontos), lengua móvil y protruible, coronada con una cresta dorsal que se extiende desde la cabeza hasta la cola, que en los machos puede llegar hasta $3 \mathrm{~cm}$ de altura, en las hembras es más pequeña; posee un pliegue angular no dilatable en el cuello, es una especie pentadáctila, con uñas en el extremo de los dedos, con propiedades prensiles que le permiten asirse a las ramas de los árboles (FONTANILLAS et al., 2000), presenta, además una escama circular timpánica (VALENZUELA, 1981).

Se le localiza desde el norte de México hasta América del Sur (GARZA-CASTRO, 1998), el sureste de Brasil (HYATT, 2003) e Islas Caribeñas del Pacífico y Antillas (ALVARADO Y SUAZO, 1996). Altitudinal mente ve desde 0 hasta $1.200 \mathrm{msnm}$ (LÓPEZ-BRIONES, 1992).

Iguana iguana es un importante recurso en la economía de muchas familias latinoamericanas con patrones culturales muy definidos acerca de la venta y consumo de carne y huevos de este reptil y dónde a éstos últimos se les atribuyen propiedades afrodisiacas debido a su alto contenido de proteína y esto ha llevado a la población al usufructo indiscriminado de la especie, generando una disminución importante no sólo en la población sino también en su entorno (DEL CAMPO PARRA, 1986; HARRIS 1982; LEGUÍZAMO, 1987 citados POR PETERS, 1993). Desafortunadamente el desconocimiento de estos productos y la escasa información acerca de ellos, sin duda alguna, han llevado a la población al consumo indiscriminado de los huevos por lo que debe llegar a diezmar considerablemente la población de cualquier especie que enfrente este mismo problema.

Sumado a esto, la implantación de tecnologías productivas sin el conocimiento necesario para la preservación de la especie, como lo puede ser la caracterización y conformación de los huevos, tema que hasta el momento no había sido tratadohan generado resultados inciertos a la hora de llevarlas a la práctica.

Además, la comercialización de los diferentes productos, las alteraciones de sus hábitats y la creciente caza furtiva, la han llevado a su condición de especie con alguna amenaza CITES - Convención Sobre el Comercio Internacional de Especies Amenazadas de Fauna y Flora.

La información presentada se basa en la caracterización y conformación del huevo, como el color, determinación de las características morfométricas, gravedad específica, porcentaje de cáscara y grosor de la misma.

También la determinación química como cantidad y calidad del albumen denso, cantidad de Calcio y Fósforo inorgánicos en cáscara, proteína total, colesterol total y grasa cruda, todos ellos ejes fundamentales para la perpetuidad de la especie y para que de esta manera se puedan programar proyectos adjuntos, enfocados a la recuperación, manejo y aprovechamiento sostenible y por supuesto racional, con fines sociales y ambientales de una especie promisoria susceptible de explotación zootécnica (GONZÁLEZ, 1993).

\section{MATERIALES Y MÉTODOS}

Material de estudio: La recolección del material biológico se realizó de dos nidos, diez huevos de cada uno de ellos, en la hacienda Brasilia, Km. 19 vía Girardot a 80 $\mathrm{Km}$. de Bogotá, ubicada en el departamento de Cundinamarca, municipio de Tocaima, vereda Pubenza situada a 400 m.s.n.m y con temperaturas anuales promedio entre $20^{\circ} \mathrm{C}$ y $24^{\circ} \mathrm{C}$. 
Determinación de las variables físicas: Los huevos se pesaron individualmente en una Balanza analítica SARTORIUS Basic modelo BA $110 \mathrm{~S}$ de 110,0000 gramos con precisión de $0.0001 \mathrm{~g}$. La prueba de color se realizó Por el método de espectrofotocolorimetria - colorímetro de sólidos (ColorTec PCM/PSM color meter) utilizando el sistema desarrollado por la Comisión Internacional de Luminosidad (CIE 1986). Las medidas del huevo, diámetro longitudinal y diámetro Transversal se tomaron con un calibrador con precisión de $0,05 \mathrm{~mm}$. El largo y ancho perimetrales con cinta métrica flexible. La determinación de la gravedad específica (ge) por el método de flotación en soluciones salinas. El porcentaje de cáscara por la fórmula: $\%$ de cáscara $=$ peso del cascarón $/$ peso del huevo * 100 . El grosor de la cáscara por medio del micrómetro (MOORE and WRIGTH) de $\pm 0,01 \mathrm{~mm}$ y sin error de cero. $(0,01 \mu \mathrm{m})$. La resistencia de la cáscara a la rotura se determinó en un Flexómetro Texturómetro E2 Test (Shimadzu CAP 500 N). Mordaza (Shearing Stress E2 - Test) (SEE) celda de carga $100 \mathrm{~N}$.

Análisis químico: Estas variables en su orden se determinaron con las siguientes metodologías: Determinación de calcio en cenizas por método complexométrico EDTA 0,01 N. Fósforo inorgánico Método colorimétrico con molibdovanadato de amonio. (A.O.A.C, 1984). Proteína total por el método Kjeldahl (A.O.A.C, 1984). Determinación de grasa cruda por medio del extractor de grasa por ciclos. (A.O.A.C, 1984).

Análisis estadístico: Se utilizó estadística descriptiva, para estimar medidas de tendencia central y dispersión, caracterizar cada una de las variables trabajadas y verificar la validez estadística de todos los datos tomados.

Para todas las variables en estudio se aplicó un análisis univariante para definir si bajo el nivel de confiabilidad del $95 \%$ se encontraba diferencia significativa entre los dos nidos o muestras según correspondan; También, se aplicó un análisis para hallar el valor de correlación y determinar el comportamiento de una variable respecto a la otra o si una característica del huevo influye en la otra positiva o negativamente. Por último, se calculó mediante regresión lineal, modelos predictores que permitieron estimar bajo un nivel de confianza del 95\% el valor de una variable o característica como dependiente a partir de otra(s) como independiente(s).

\section{RESULTADOS Y DISCUSIÓN}

Se registran los pesos de los huevos (en gramos), de cada uno de los dos nidos. Estos datos fueron tomados al día siguiente de haber sido extraídos del medio natural y de haber llevado a cabo un análisis descriptivo, donde se halló que el promedio general de peso de los huevos está en 14,78 gramos con un error típico de 0,18 gramos, indicando que bajo el coeficiente de variación de 5,58\% los datos son homogéneos y bajo un nivel confianza del $95 \%$ se puede concluir que el peso de los huevos de la especie Iguana iguana oscilan entre 14,39 y 15,16 gramo y se encuentran dentro del rango de 12 a 18 gramos reportado por MENDELSSOHN (1980) y MILLER (1987) y también con los datos reportados por DE LA OSSA (1995), quién propone un peso entre 12,91 y $15,5 \mathrm{~g}$, con la única diferencia de que el intervalo del promedio es más corto en los datos obtenidos en este trabajo.

Con respecto al color, los datos obtenidos por medio del espectrofotocolorímetro (ColorTEC-PCM) no se analizan ya que el equipo viene programado para arrojar esta información. El color de la cáscara de los huevos de Iguana se puede clasificar entre amarillo medio y blanco amarillento.

Diámetro longitudinal, de los 20 huevos analizados en total se obtiene un promedio del diámetro longitudinal en milímetros de $39,705 \pm 0.49$ y no se encontraron diferencias estadísticamente significativas $(P>0.05)$ entre los datos.

Diámetro transversal, el promedio general del diámetro transversal en $\mathrm{mm}$ es de 26,4 $\pm 0,6$; dando un intervalo de confianza al 
Guzmán et al - Estudio físico-químicos de los huevos de Iguana iguana Linnaeus, 1758.

95\% de 25,0 a 26,9 mm; Mientras que lo reportado por MENDELSSOHN (1980) y MILLER (1987) es de 21 a $28 \mathrm{~mm}$ de diámetro y el de DE LA OSSA (1995) es de 22 a $28 \mathrm{~mm}$ con un promedio de $23,5 \mathrm{~mm}$.

Largo perimetral, los huevos de la especie en estudio tienen un promedio total de $11,5 \pm 0.2 \mathrm{~cm}$. Esto se corrobora con un Coeficiente de Varianza (CV) total de $3,81 \%$ y bajo el $95 \%$ de confianza se puede afirmar que el largo perimetral de los huevos de Iguana tiene un intervalo de 11,34 a $11,76 \mathrm{~mm}$ de largo siendo este un estimativo para la especie ya que no se encuentran parámetros ni estudios publicados para compararlos.

Ancho perimetral, los huevos analizados presentan un promedio de $9.0 \pm 0,2$ $\mathrm{cm}$., con un intervalo de confianza de 8,9 a $9,1 \mathrm{~cm}$. No se han encontrado hasta el momento referencias bibliográficas al respecto.

Gravedad específica, la gravedad específica (GE) promedio para los huevos de esta muestra, es de 1,0725 $\pm 0,002 \mathrm{~g} / \mathrm{Cm} 3$, con un Coeficiente de Variación (CV) de $0,46 \%$. Esto indica que la media obtenida es confiable por la homogeneidad de los datos y que bajo un $95 \%$ de confiabilidad, el rango para gravedad específica es de 1,070 a $1,074 \mathrm{~g} / \mathrm{Cm} 3$.

Porcentaje de cáscara, con un promedio total de 3,05 $\pm 0,15 \%$, y un CV de $9,75 \%$. Los datos encontrados en el porcentaje de cáscara de huevos de la especie en estudio son homogéneos. Hasta el momento de la especie en estudio no se han encontrado datos acerca de esta variable.

Grosor de cáscara en el polo ancho, la medida para el grosor de la cáscara en huevos de Iguana iguana es de 105,33 \pm 3,09 $\mu \mathrm{m}$, con una varianza de 5,81\%; determinando de esta manera que los datos encontrados para esta variable se comportan homogéneamente.

Grosor de la cáscara en el polo angosto, el promedio general es de 102,78 $\pm 2,15 \mu \mathrm{m}$, con un coeficiente de variación de 9,90\%, de lo cual se deduce que su grado de homogeneidad es moderado. El intervalo de confianza al $95 \%$ para esta media se estima en $98,01 \mu \mathrm{m}$ y $107,55 \mu \mathrm{m}$.

Grosor de la cáscara en la zona ecuatorial 1 , el promedio general para esta variable

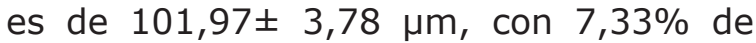
coeficiente de varianza; implicando que los datos se encuentran moderadamente homogéneos y que bajo el $95 \%$ de confianza, se puede calcular que el promedio para el grosor de la cáscara en la zona ecuatorial 1 se encuentra en un rango de $98,46 \mu \mathrm{m} \mathrm{a}$ $105,46 \mu \mathrm{m}$.

Grosor de la Cáscara en la zona ecuatorial 2, para esta variable se tiene como resultado una media de $102,59 \pm 4,10 \mu \mathrm{m}$ y un CV de $7,9 \%$. Esto indica que los datos están un poco dispersos alrededor de la media general y que por tanto tienen una fuente de variación.

La determinación del grosor de la cáscara es muy importante al igual que el punto de medición ya que la cáscara no es uniformemente gruesa. El grosor es mayor en el polo fino, intermedio en el polo grueso y menor en el ecuador. En huevos de gallina el grosor de cáscara como media, está comprendido entre $0.28 \mathrm{~mm}$ y 0.42 mm (BUXADÉ, 2000).

Resistencia a la rotura, los resultados están expresados en medidas de Newton por milímetro $(\mathrm{N} \times \mathrm{mm})$, Newton $(\mathrm{N})$ y Newton por milímetro cuadrado $(\mathrm{N} \times \mathrm{mm} 2)$ y son un estimativo de cómo es la resistencia a la rotura de la cáscara en los huevos de la especie en estudio. En promedio para romper la cáscara del huevo de Iguana iguana se necesita una fuerza de 15,97 $\pm 6,65 \mathrm{~N}$, donde para un $\mathrm{mm} 2$ se debe aplicar 1,43 $\pm 1,78 \mathrm{~N}$. por lo tanto, para el límite de confianza al 95\% con estos datos, se tiene un intervalo de 0,62 a 1,98 $\mathrm{N}$ por $\mathrm{mm} 2$ (Figura 1) 


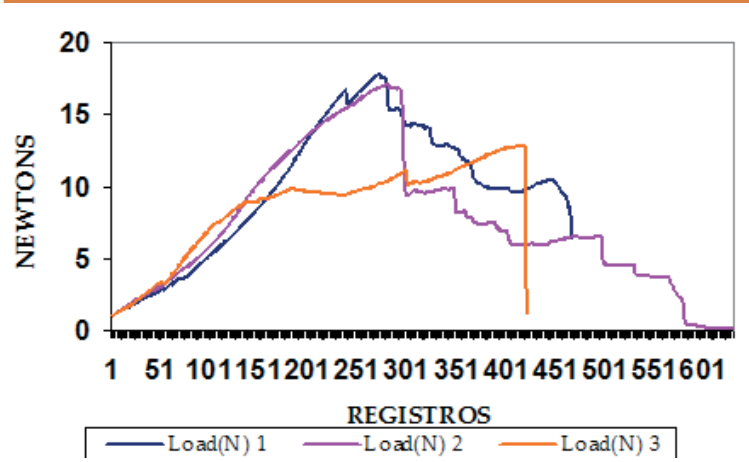

Figura 1. Prueba de rotura para cada una de las muestras expresadas en $\mathrm{N}$.

En cuanto a las variables químicas, se tiene:

Calcio Inorgánico, para los huevos de iguana, la cáscara tiene un 51,235 $\pm 3,02$ $\%$ de calcio inorgánico. Fósforo Inorgánico en Cáscara, el porcentaje promedio de Fósforo inorgánico (Pi) en cáscara de huevos de Iguana de esta muestra es de $20,170 \pm 1,56 \%$. Proteína total por el método de Kjeldahl, $51,47 \pm 1,05 \%$ de proteína total en el huevo de Iguana iguana de esta muestra, es el promedio general calculado bajo los datos arrojados por el método de Kjeldahl.

Grasa cruda, el porcentaje de grasa cruda en promedio es de $17,020 \pm 1,33 \%$. Albumen compacto, el promedio general para el albumen compacto expresado en gramos es de $3,8713 \pm 0,21 \mathrm{~g}$.

\section{CONCLUSIONES}

La caracterización cromática del huevo de la especie en estudio determinó que se pueden clasificar entre amarillo medio y blanco amarillento. Los huevos de la especie Iguana iguana $(\mathrm{n}=20)$ tienen como características físicas en promedio un peso de 14,78 gramos; un diámetro longitudinal de $39,70 \mathrm{~mm}$; diámetro transversal de $26,41 \mathrm{~mm}$; largo perimetral de $11,55 \mathrm{~cm}$; ancho perimetral de 9.07 $\mathrm{cm}$; una gravedad específica de $1,072 \mathrm{~g} /$ $\mathrm{Cm} 3$; porcentaje de cáscara del 3,04\%; y un grosor en cada uno de sus polos así: polo ancho de $105,33 \mu \mathrm{m}$; polo angosto de $102,78 \mu \mathrm{m}$; zona ecuatorial 1 de $101,96 \mu \mathrm{m}$; zona ecuatorial 2 de 102,60 $\mu \mathrm{m}$ y una resistencia a la rotura de 1,43 $\mathrm{N} \times \mathrm{mm} 2$. Tanto el peso como el grosor en las zonas ecuatoriales 1 y 2 presentan diferencias estadísticas $(P<0,05)$ entre nidos.

En la composición química de los huevos de la Iguana verde ( $n=4$ muestras) se encontró que éstos tienen en cáscara de $51,23 \%$ de Calcio Inorgánico; 20,17\% de Fósforo Inorgánico; en albumen un $51,47 \%$ de proteína bruta y un $17,02 \%$ de grasa cruda y en el albumen compacto un peso de 3,87 gramos.

Existe correlación negativa entre el diámetro transversal y la energía necesaria para romper la cáscara de los huevos, entre largo perimetral y gravedad específica, peso y porcentaje de cáscara; es decir, entre el tamaño y la calidad del huevo.

Medianteregresiones múltiplessedeterminó que el peso depende del porcentaje de cáscara y del albumen compacto en un $87 \%$, la gravedad específica depende no solo del largo perimetral sino también del porcentaje de cáscara y del grosor de ésta en la zona ecuatorial en un $86,3 \%$ y el porcentaje de cáscara, del peso, gravedad específica y grosor en la zona ecuatorial en $88,3 \%$.

La resistencia a la rotura de la cáscara depende del porcentaje de fósforo inorgánico en un $99,7 \%$, el calcio inorgánico depende del grosor de la cáscara y del peso del huevo en un $99,9 \%$ y el albumen compacto depende del peso y del ancho perimetral en un $99,9 \%$.

\section{Agradecimientos}

A la Universidad De Cundinamarca por brindarnos la oportunidad de desarrollar este trabajo, CORPOICA Tibaitatá por su valioso aporte en los análisis de color y rotura de la cáscara, Dr. John Alexander Moreno y al Dr. Jairo Enrique Granados por su invaluable colaboración y gran conocimiento para la culminación de este trabajo, a la Doctora Herly Zúñiga, a Eliana Torres y Alberto Suárez por su contribución en el trabajo de laboratorio, los Doctores Luís Miguel Acosta y Vilma Moreno por su inmenso apoyo, a todos los que colaboraron de una u otra forma para que este trabajo saliera adelante y a usted por destinar un poco de su tiempo para leer este trabajo. 


\section{REFERENCIAS}

ALVARADO, D.J.; SUAZO, O.I. 1996. Las iguanas de México. Historia Natural y Conservación. México: Laboratorio Tortuga Marina y Biología de la Conservación. Facultad de Biología.

A.O.A.C. 1984. Oficial Methods Of Analysis Of The Association of Official Analytical Chemist Centennial Edition, Eggs and Egg Products. Edited by Sidney Williams. Fourteenth edition. Virginia U.S.A.

BUXADÉ, C.C. 2000. La gallina ponedora. 2a Edición. Ediciones Mundi-Prensa. Madrid, España.

CITES. 1988. Significant trade in wildlife a review of selected species. CITES Appendix II, Volume 2: Reptiles and invertebrates. Cambridge.

CITES. 2012. Publication on iguanas. Convention on International Trade in Endangered Species

Of Wild Fauna and Flora. Switzerland. Disponible en: http://www.cites.org/common/com/ ac/26/E26-04i.pdf. Consultado: 06-09-2017.

DEL CAMPO PARRA, A. 1986. Uso y manejo tradicional de la fauna Silvestre y sus relaciones con otras actividades productivas en San Pedro Jicayan, Oaxaca. Xalapa, Veracruz, México. Cuadernos de divulgación INIREB Nº 27.

DE LA OSSA, J.V. 1995., Estudio ecológico sobre reproducción de Iguana iguana (Linnaeus, 1758) y su utilización como recurso alimenticio en San Marcos (Sucre) Colombia. Trabajo de Grado. 158 pág. Mimeografiado. Universidad Incca de Colombia. Bogotá.

FONTANILLAS, P.J.C.; GARCÍA, A.C.; GASPAR, S.I. 2000. Los Reptiles. Biología, Comportamiento y Patología. Ediciones Mundi-Prensa. $1^{a}$ Edición. España.

GARZA-CASTRO, J.M. 1998. Dieta en crías de Iguana iguana en cautiverio. Memorias del Primer taller nacional sobre manejo de iguanas en cautiverio. Pátzcuaro (Michoacán) México. Subcomité Técnico Consultivo para el Manejo y Aprovechamiento Sustentable de las iguanas en México.16-18.

GONZALEZ, A. 1993. Proyecto Iguana Verde. División de Desarrollo Sostenible de ANCON. Edición Junio 1993. Panamá.

HARRIS, D.M. 1982. The phenology, growth and survival of the green iguana (Iguana iguana) in Northern Colombia. Págs. 150-161. En: Burghardt, G.M.; RAND, A.S. (eds.). Iguanas of the world; their behavior, ecology and conservation. Noyes Publ. Park Ridge, N.J.

HYATT, J. 2003. La iguana. Tesoro nacional en peligro de extinción. México desconocido 318:1-3.

LEGUIZAMO, I. 1987. Plantas leñosas alimento de la iguana. Montería, Universidad de Córdoba - ICFES.

LÓPEZ-BRIONES, F. 1992. Iguana verde (Iguana iguana). Facultad de Medicina Veterinaria y Zootecnia. Tesis de licenciatura. UNAM. México. 
MENDELSSOHN. H. 1980. Observations on a captive colony of Iguana iguana. P+gas. 119123. En: MURPHY, J.B.; COLLINS, J.T. (eds.). Reproductive biology and diseases of captive reptiles. SSAR Contributions to Herpetology, volume 1.

MILLER, T. 1987. Artificial incubation of eggs of the green iguana (Iguana iguana). Zoobiology (6) 225-236.

PETERS, H. 1993., La Iguana verde (Iguana iguana) potencialidades para su manejo. Documento Técnico. Santiago de Chile. FAO, Oficina regional para América Latina y el Caribe y FAO/PNUMA.

VALENZUELA, L.G. 1981. Contribución al conocimiento de la biología y ecología de Ctenosaura pectinata e Iguana iguana (Reptilia: Iguanidae) en la costa de Jalisco. Facultad de Ciencias. Tesis de Maestría UNAM. México. 